\section{Coupled Twin Slots Fed by Conductor-Backed Coplanar Waveguide}

I-Ching Lan, Shih-Yuan Chen, and Powen Hsu

\begin{abstract}
The coupling mechanism of the twin slots fed by the conductor-backed coplanar waveguide is investigated. The goal is to improve the antenna efficiency by using the phase cancellation technique and at the same time keep the structure as simple as possible. The leakage phenomenon is illustrated visually and the effects of the antenna parameters are examined numerically for choosing the appropriate variables. Finally the proposed antenna is fabricated and tested, showing $69.8 \%$ of antenna efficiency.
\end{abstract}

Index Terms-Antenna feeds, coplanar waveguides, parallel plate waveguides, slot antennas.

\section{INTRODUCTION}

Coplanar-waveguide-FED slot antennas are attractive due to their uniplanar structures and the ease of fabrication [1]. To render the radiation from bidirectional to unidirectional, conducting planes are usually placed at the back of the antennas. The performance, however, suffers because the conductor-backed coplanar waveguide (CBCPW) and the conductor-backed slot are both leaky structures due to the incursion of the parallel-plate mode leakage [2]. The parallel-plate TEM mode has zero cutoff frequency, so the leakage phenomenon occurs at all frequencies. One major trend toward solving the leakage problem of conductorbacked slots is the phase cancellation technique originally proposed in the 1980s to deal with the surface wave problem of dipoles [3] as well as slots on electrically thick dielectric substrates [4] using infinitesimal elements. This technique uses twin broadside slots half a guided wavelength apart to cancel the undesired propagating power. Successful implementations of this technique for conductor-backed slots can be found in [5] and [6], where the slot lengths are 0.84 and 0.95 wavelengths long in [5] and about one wavelength long in [6]. For these excellent works, the complexities always come from the feeding structures, since both slots are fed directly and separately. Two dielectric layers and feeding circuits are used in [5], whereas two dielectric layers and two feeding ports are required in [6].

In this paper we propose a new feeding mechanism for the twin slots configuration, of which the first slot dipole is directly fed by the CBCPW, whereas the second slot is coupled by the parallel-plate mode leakage excited at the discontinuity of the first slot dipole. By means of this feeding method, the resulting geometry is single-layered with only one feed and without any additional feeding circuits or via holes. The simplicity and conciseness greatly enhance the usability of CBCPW-fed slot antennas for practical applications.

\section{Antenna Structure And Design}

The single slot dipole fed by the CBCPW acts more like a mode converter than an efficient antenna. It converts most of the power from the

Manuscript received November 30, 2007; revised March 1, 2008. This work was supported by the National Science Council, Taiwan, R.O.C., under Contract NSC 96-2752-E002-002-PAE.

I-C. Lan and S.-Y. Chen are with the Department of Electrical Engineering and the Graduate Institute of Communication Engineering, National Taiwan University, Taipei 10617, Taiwan, R.O.C. (e-mail: sychen@cc.ee.ntu.edu.tw).

P. Hsu is with the Department of Electrical Engineering, the Graduate Institute of Communication Engineering, and the Graduate Institute of Photonics and Optoelectronics, National Taiwan University, Taipei 10617, Taiwan, R.O.C. (e-mail: phsu@cc.ee.ntu.edu.tw).

Digital Object Identifier 10.1109/TAP.2008.923342

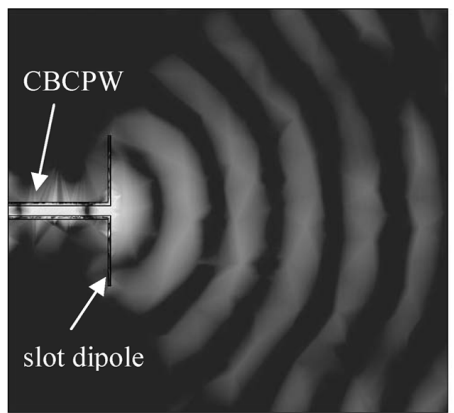

(a)
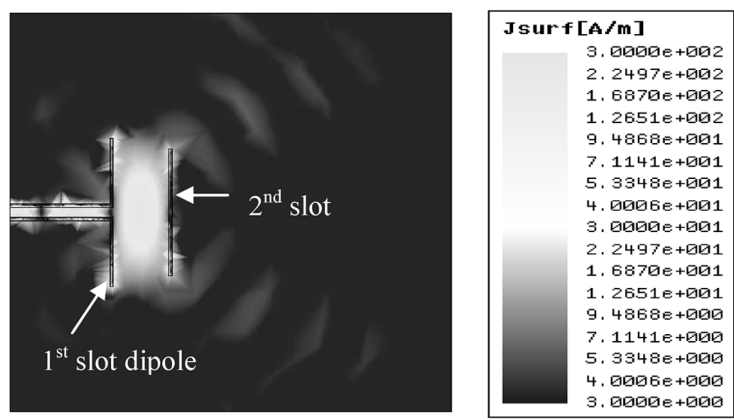

(b)

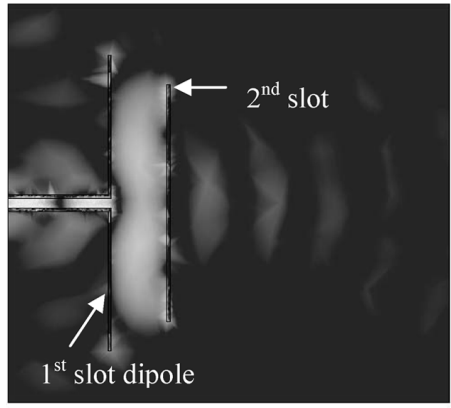

(c)

Fig. 1. Simulated surface current density distributions of (a) a single slot dipole, (b) twin slots with the first slot dipole $\sim 1 \lambda$ long, and (c) twin slots with the first slot dipole $\sim 2 \lambda$ long. All cases are fed by the CBCPW with the same dimension.

CBCPW mode to the parallel-plate mode, leaving the radiated power very small. The typical antenna gain is far below $0 \mathrm{dBi}$ and the radiation efficiency below $10 \%$. Using the simulator HFSS from Ansoft, Fig. 1(a) illustrates this phenomenon, where the surface current density at the interface between the dielectric and the top metal layer is plotted. At the junction of the CBCPW feed-line and the slot dipole, the parallel-plate mode is strongly excited and propagates radially into the substrate region. In contrast, as Fig. 1(b) and (c) show, if an additional slot with suitable dimension is placed in front of the first slot dipole at a distance about half a guided wavelength of the parallel-plate mode according to the phase cancellation technique, the leakage is reduced significantly. Although the leakage suppression is not complete, the gain and the efficiency are greatly improved, as will be shown shortly.

The geometry and the associated parameters of the proposed twin slots fed by the CBCPW are shown in Fig. 2. The gray area represents the metal portion and the white ones are the etched slots and the feedline. The relative phases of the fields on the first and the second slots depend on the distance $d$ as well as the lengths and widths of the slots, $L_{1}, L_{2}, S_{1}$, and $S_{2}$. When the distance $d$ is half a guided wavelength of 


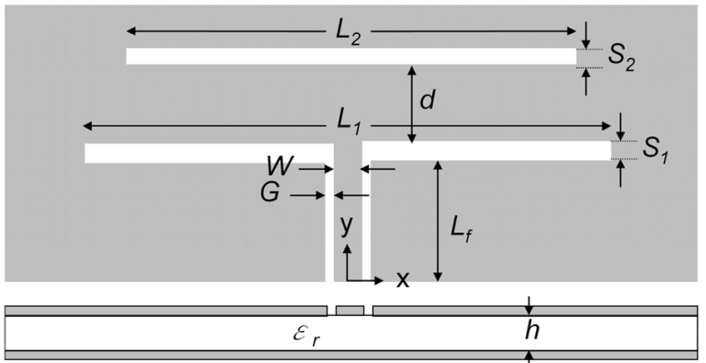

Fig. 2. Geometry of the proposed twin slots configuration.

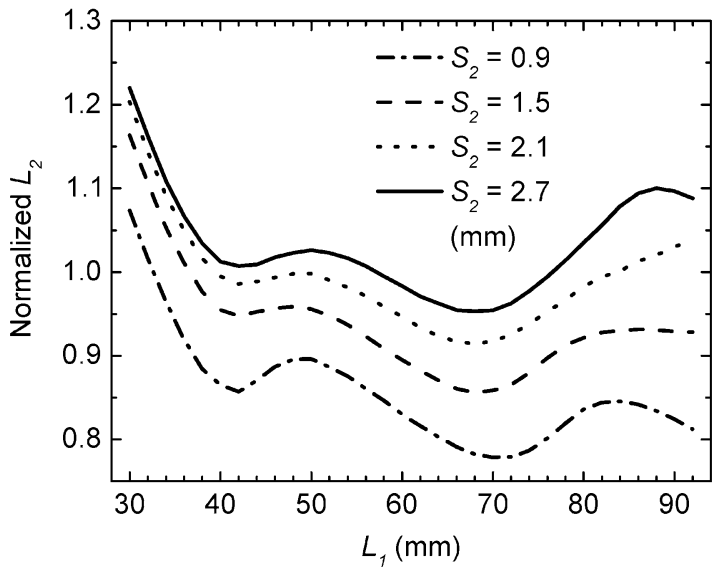

Fig. 3. Simulated results for normalized $L_{2}$ versus $L_{1}$ with a broadside main beam at $5 \mathrm{GHz} . S_{1}=1.5 \mathrm{~mm}, L_{f}=15 \mathrm{~mm}, d=13.1 \mathrm{~mm}, W=2.5 \mathrm{~mm}$, $G=0.8 \mathrm{~mm}, h=1.6 \mathrm{~mm}, \epsilon_{r}=4.2$, and $\tan \lambda=0.02$.

the parallel-plate mode according to the phase cancellation technique and the slots are of the same length, it is found that usually the phases are not exactly equal, resulting in an off-broadside main beam in the E-plane (y-z plane). The phases can be made equal if the slot lengths are allowed to be discrepant. Thus for each $L_{1}$ value, we can find a corresponding $L_{2}$ that results in a broadside main beam, assuming that the transverse dimensions ( $x-y$ plane) of the substrate and the ground are infinite so the influence of the edge diffractions on radiation patterns is temporarily ignored. The following simulations are carried out using the package software IE3D from Zeland. Fig. 3 illustrates the simulated results at $5 \mathrm{GHz}$, where the normalized $L_{2}\left(L_{2} / L_{1}\right)$ is plotted versus $L_{1}$. The effect of different slot widths is also considered. From Fig. 3 we see that $L_{2}$ might be greater than, equal to, or smaller than $L_{1}$, depending on the choices of $L_{1}$ and the widths of the slots. Note that since the CBCPW is a leaky line, the length of the feed-line $\left(L_{f}\right)$ should not be too long. In our simulations the feed-line length is chosen to be $15 \mathrm{~mm}$, with the corresponding loss estimated to be $0.45 \mathrm{~dB}$.

For each combination of $L_{1}$ and $L_{2}$ that results in a broadside main beam, the corresponding efficiencies and gains are calculated and shown in Fig. 4. Here the radiation efficiency is defined as the ratio between the radiated power and the input power, whereas the antenna efficiency as the ratio between the radiated power and the incident power. The difference between the incident power and the input power is the return loss at the input. Fig. 4(a) reveals that the radiation efficiency has local maximums when $L_{1}$ is near integer multiples of $\lambda$, where $\lambda$ is approximated by $\lambda_{0} / \sqrt{\left(\varepsilon_{r}+1\right) / 2}$, which is $37.2 \mathrm{~mm}$ at 5 $\mathrm{GHz}$ for the present case. However, the serious input mismatch makes the overall antenna efficiency below $50 \%$ when $L_{1} \fallingdotseq \lambda$. On the other hand, the highest antenna gain is achieved when $S_{2}=2.7 \mathrm{~mm}$ and $L_{1}=78 \mathrm{~mm} \fallingdotseq 2 \lambda$, as can be seen in Fig. 4(b). The corresponding

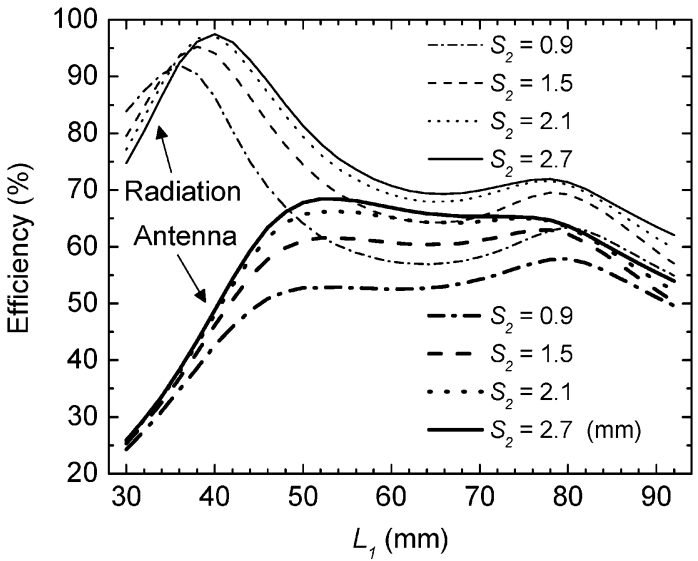

(a)

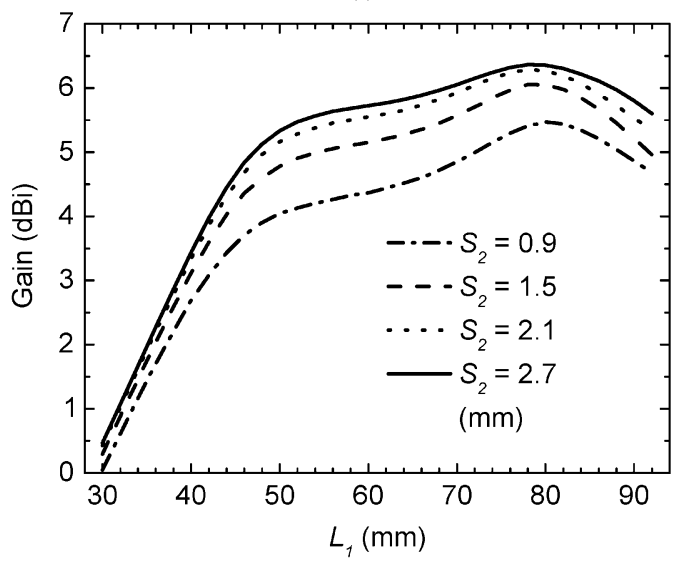

(b)

Fig. 4. Simulated (a) radiation and antenna efficiencies and (b) antenna gain versus $L_{1}$ at $5 \mathrm{GHz} . S_{1}=1.5 \mathrm{~mm}, L_{f}=15 \mathrm{~mm}, d=13.1 \mathrm{~mm}, W=2.5$ $\mathrm{mm}, G=0.8 \mathrm{~mm}, h=1.6 \mathrm{~mm}, \epsilon_{r}=4.2$, and $\tan \lambda=0.02$.

antenna efficiency is $64.7 \%$ but the input return loss is just about 10 $\mathrm{dB}$. If a better input matching condition is required, smaller $L_{1}$ values should be chosen such that the curves of the radiation and antenna efficiencies are closer to each other. In our experiment we choose $L_{1}=60 \mathrm{~mm}$ for a better input return loss and a slightly higher antenna efficiency, although the gain is smaller due to the smaller antenna size. Note that although it seems like increasing $S_{2}$ results in higher efficiencies and gains, these performances soon begin to saturate and finally fall off. Therefore in our simulations higher $S_{2}$ values are not pursued further.

\section{EXPERIMENTAL RESULTS}

A twin slot fed by the CBCPW is fabricated and tested with the following parameters: $L_{1}=60 \mathrm{~mm}, L_{2}=59 \mathrm{~mm}, S_{1}=1.5 \mathrm{~mm}$, $S_{2}=2.7 \mathrm{~mm}, d=13.1 \mathrm{~mm}, L_{f}=15 \mathrm{~mm}, W=2.5 \mathrm{~mm}$, and $G=0.8 \mathrm{~mm}$. The FR4 substrate with $h=1.6 \mathrm{~mm}, \varepsilon_{r}=4.2$, and $\tan \delta=0.02$ is used in the fabrication. Note that for antenna applications, the substrate material with lower dielectric constant and loss tangent would be more preferable. However, in this work, the FR4 substrate was used merely because it is more accessible to us and much cheaper than other substrates. The dimensions of the substrate and the ground plane are $150 \mathrm{~mm}$ in the $\mathrm{x}$-direction and $85 \mathrm{~mm}$ in the y-direction.

Fig. 5 plots the frequency response of the simulated and measured input return losses and gains. The measured input return loss is seen to have about $0.1-\mathrm{GHz}$ frequency shift from the simulated one, whereas 


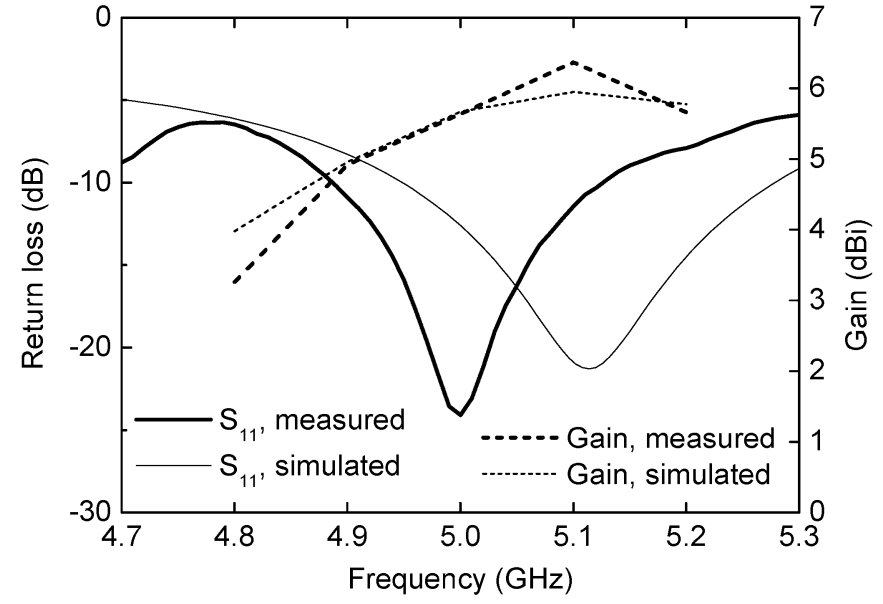

Fig. 5. Simulated and measured input return losses and gains of the proposed antenna with $L_{1}=60 \mathrm{~mm}, L_{2}=59 \mathrm{~mm}, S_{1}=1.5 \mathrm{~mm}, S_{2}=2.7 \mathrm{~mm}$, $d=13.1 \mathrm{~mm}, L_{f}=15 \mathrm{~mm}, W=2.5 \mathrm{~mm}, G=0.8 \mathrm{~mm}, h=1.6 \mathrm{~mm}$, $\epsilon_{r}=4.2$, and $\tan \lambda=0.02$.

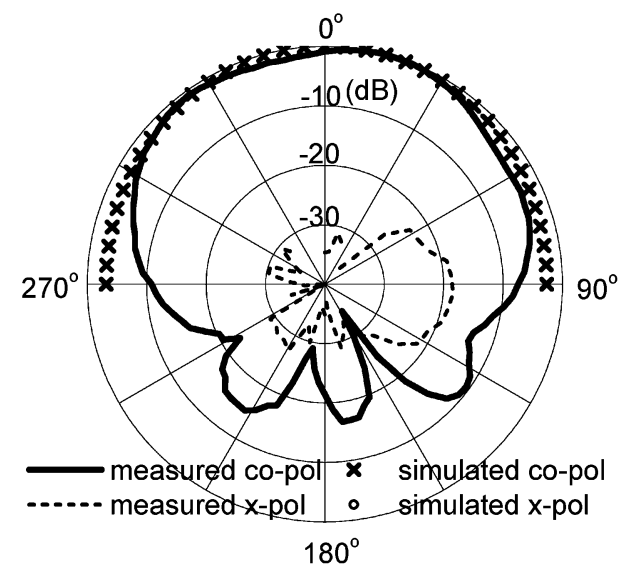

(a)

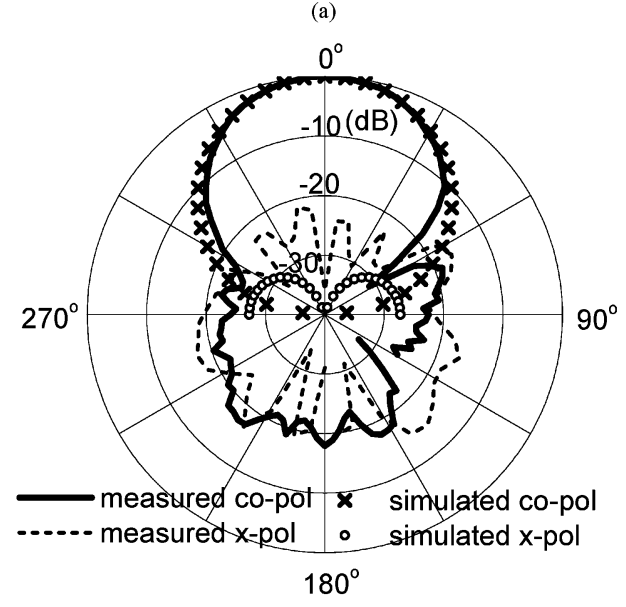

(b)

Fig. 6. Simulated and measured radiation patterns of the proposed antenna at $5 \mathrm{GHz}$. (a) E-plane (y-z plane). (b) H-plane (x-z plane).

the simulated and measured gains are very close to each other. Considering that the simulation tool IE3D does not take the finite ground and the finite substrate into account and the mechanical tolerance of the fabrication process would unavoidably result in some discrepancy between the physical dimensions of the simulation model and the test piece, the results are quite satisfactory. At $5 \mathrm{GHz}$ the measured return loss is $-24.1 \mathrm{~dB}$ and the measured gain is $5.64 \mathrm{dBi}$. The measured $10-\mathrm{dB}$ return loss bandwidth is $5 \%$, extending from $4.88-5.13 \mathrm{GHz}$. Fig. 6 shows both the simulated and measured E- and H-plane radiation patterns at $5 \mathrm{GHz}$. Mild ripples are observed in the measured E-plane co-polarization pattern. This is caused by the edge diffractions of the finite ground plane, which is a common phenomenon in slot E-planes. The measured cross-polarization levels are fairly low in the E-plane and a bit higher in the $\mathrm{H}$-plane, but are still below $-15 \mathrm{~dB}$ in all directions. Note in Fig. 6(a) that the simulated cross-polarized component in the E-plane is invisible. This is because the component remains lower than $-40 \mathrm{~dB}$, which is the lower bound of the radial axis in the figure. The measured front-to-back ratio is higher than $17 \mathrm{~dB}$.

By using the method described in [7] to estimate the directivity from the half-power beamwidths of both the E- and H-plane radiation patterns, together with the measured gain data, the antenna efficiency is found to be $69.8 \%$ at $5 \mathrm{GHz}$. For comparison, the antenna efficiency of the CBCPW-fed slot dipole with an arc-slot [8] is calculated using the same method, and is found to be $50.6 \%$. Although the structure in [8] has the advantage of occupying a smaller area, the performance of the present structure is superior in terms of the antenna efficiency, the main concern of antennas fed by the leaky CBCPW.

\section{CONCLUSION}

The CBCPW-fed coupled twin slots have been proposed and the properties demonstrated. The coupling mechanism and the effects of the antenna parameters have been studied and utilized to design a unidirectional antenna of $69.8 \%$ antenna efficiency with a simple structure without any complicated feeding circuits. This type of antennas would be very attractive when the CBCPW feed is unavoidable and the whole structure must be kept simple.

\section{REFERENCES}

[1] R. N. Simons, Coplanar Waveguide Circuits, Components, and Systems. New York: Wiley, 2001, ch. 1.

[2] H. Shigesawa, M. Tsuji, and A. A. Oliner, "Conductor-backed slot line and coplanar waveguide: Dangers and full-wave analyses," in IEEE MTT-S Int. Microwave Symp. Dig., 1988, pp. 199-202.

[3] D. M. Pozar, "Considerations for millimeter-wave printed antennas," IEEE Trans. Antennas Propag., vol. AP-31, no. 5, pp. 740-747, Sep. 1983.

[4] R. L. Rogers and D. P. Neikirk, "Use of broadside twin element antennas to increase efficiency on electrically thick dielectric substrates," Int. J. Infrared Millimeter Waves, vol. 9, no. 11, pp. 949-969, 1988

[5] M. Qiu and G. V. Eleftheriades, "Highly efficient unidirectional twin arc-slot antennas on electrically thin substrates," IEEE Trans. Antennas Propag., vol. 52, no. 1, pp. 53-58, Jan. 2004.

[6] J. P. Jacobs, J. Joubert, and J. W. Odendaal, "Radiation efficiency and impedance bandwidth of conductor-backed CPW-fed broadside twin slot antennas on two-layer dielectric substrate," Proc. Inst. Elect. Eng. Microw. Antennas Propag, vol. 150, no. 4, pp. 185-190, Aug. 2003.

[7] W. L. Stutzman, "Estimating directivity and gain of antennas," IEEE Antennas Propag. Mag., vol. 40, no. 4, pp. 7-11, Aug. 1998.

[8] I. C. Lan and P. Hsu, "Gain-enhanced slot antenna fed by conductorbacked coplanar waveguide," in Proc. 18th Asia Pacific Microwave Conf., Yokohama, Japan, 2006, pp. 73-76. 